\title{
On Using Image Processing Techniques for Evaluation of Mammography Acquisition Errors
}

\author{
Maira B. H. Moran $^{1}$, Aura Conci ${ }^{1}$, Salete de J. F. Rêgo ${ }^{2}$, Cristina A. P. Fontes ${ }^{2}$, \\ Marcelo D. Brito Faria ${ }^{3}$, Luciana Freitas Bastos ${ }^{3}$, Gilson A. Giraldi ${ }^{4}$ \\ ${ }^{1}$ Institute of Computing, Universidade Federal Fluminense (UFF) - Niterói, RJ, Brazil \\ \{mhernandez, aconci\}@ic.uff.br
}

${ }^{2}$ Radiology Department, Hospital Universitario Antonio Pedro, UFF - Niterói, RJ, Brazil

sa.rego@uol.com.br, cristinasvolinsque@gmail.com

${ }^{3}$ Policlínica Piquet Carneiro, UERJ - Rio de Janeiro, RJ, Brazil

mdanbf@yahoo.com, lucianafreitasbastos@yahoo.com

${ }^{4}$ Laboratório Nacional de Computação Científica - Petrópolis, RJ, Brazil

gilsonelncc.br

\begin{abstract}
Mammography is an extremely important examination considering the high incidence of breast-related diseases, since it helps to detect several abnormalities. Errors in the acquisition can mask potential problems. The objective of this study is to apply image processing techniques for automated detection of some very common errors. Craniocaudal $(C C)$ and mediolateral oblique (MLO) mammograms views were evaluated considering aspects as: (1) breast symmetric positioning, (2) adequate nipples profiling and centering, and (3) properly pectoral muscle location. The image processing techniques used are based on: skeletonization, Hough transform and thresholding. The achieved results are impressive especially in the determination of the nipple position.
\end{abstract}

Resumo. A mamografia é um exame extremamente importante, considerando a alta incidência de doenças relacionadas à mama, uma vez que ajuda a detectar várias anormalidades. Erros na aquisição podem mascarar possíveis problemas. O objetivo deste estudo é aplicar técnicas de processamento de imagens para detectar automaticamente alguns erros muito comuns. As visualizações dos mamogramas craniocaudais (CC) e oblíquos mediolaterais (MLO) foram avaliadas considerando aspectos como: (1) posicionamento simétrico da mama, (2) correto perfilamento e centralização dos mamilos e (3) localização adequada do músculo peitoral. As técnicas de processamento de imagem utilizadas baseiam-se na esqueletização da Morfologia Matemática, transformação de Hough e limiarização após uma etapa de pré-processamento. Os resultados obtidos são muito bons, especialmente na determinação da posição do mamilo.

\section{Introduction}

Considering that breast cancers present high incidence in women, mammography is an important tool to their diagnostic process, since it allows to analyze from very small mi- 
crocalcifications to masses. However several issues can appear during the exam acquisition. Studies show that nipple improper positioning is very common in mammograms, so as others positioning failures [Popli et al. 2014]. These incorrect locations prevent proper image acquisition and adequate report of the finds [Li et al. 2010, Bassett et al. 1993]. Anatomical or pathological reasons can be the cause of such a unsuitable emplacement. It can also be caused by improper acquisition due to miss attention during the breast's placement in the device. For example, when a patient moves during the exam, the breast change its stance and consequently some problem may be hidden on the resultant mammogram.

Most of the errors in breast positioning are operator dependent, so it can be avoided if some principles are observed during acquisition [Popli et al. 2014]. The objective of this study is to apply image processing techniques that could aid in the detection of placements errors that prejudice mammograms. The main idea is to apply the methods proposed here in a previous analysis before the patient exposition to x-ray.

\section{Mammograms}

Mammograms, like conventional radiographs, use of X-rays, which focus on the breast compressed in the device. Part of these X-rays passes through the tissues and are recorded in the form of an image, using the device receptors. Since the various structures in the breasts have different densities, X-rays pass through them differently. As a result, we have images in which regions can be identified by their tonal intensity [INCA 2018].

Normally, for each breast a mediolateral oblique (MLO) and a craniocaudal (CC) view are obtained (Fig. 1). The $\mathrm{CC}$ incidence includes the entire mammary gland, lateral and medial portion, except the axillary portion [INCA 2018]. X-rays are applied head to feet. The MLO incidence allows better identification of the tissue close to the chest wall and axillary tail, in addition to better include all breast tissue [INCA 2018]. It is so named because of the compression plane used.

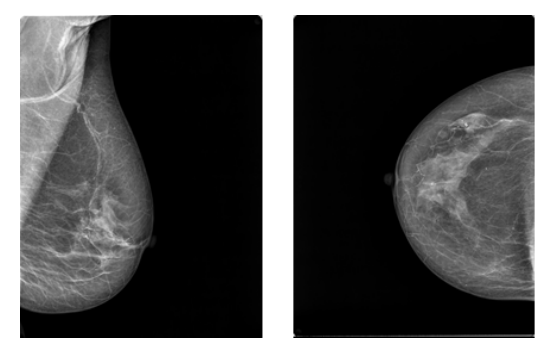

Figura 1. MLO (left) and CC (right) digital mammogram

Even thought that mammograms are the one of most appropriate breast examination tools, there are some special cases that demand extra attention during the examination evaluation. They refer to patients that present one of the following conditions: very small or masculine breast, breast implants, mastectomy, reconstructed breast, and breast-conserving surgery.

\subsection{Acquisition errors}

The main goal in mammograms is to obtain images with maximum relevant information about the breast, considering the possibility of diseases and patient comfort during the 
acquisition. There are several factors to consider, which can affect the quality of the obtained images. One of them is failures on the placement of the breast before compression by the plates of the device for the examination process. The correct location of the breast allows maximum quality of the image for analysis and findings by the radiologists. In this section we describe some of the most frequent acquisition error in mammograms.

\subsubsection{Profiled and centered nipple}

In the processes of mammogram acquisition the nipple should be positioned properly to be well identified on the image, otherwise it may be confused with other structures of the breast, which may hide retroareolar nodules [March et al. 2013]. Another point to be observed in mammograms is whether the nipple is centered. When the nipple is not centered, part of the breast may not be presented in the image to be analyzed [Popli et al. 2014].

\subsubsection{Pectoral muscle position}

Although the pectoral muscle is not part of the breast anatomy, in mammograms it helps in detection of axillary nodules and in the correct positioning of the breast, indicating if most of it is properly included on the image. The lower edge of pectoral muscle should be at the level of the nipple. In the MLO view, it is very difficult to properly capture the pectoral muscle, especially for large or obese patients [Popli et al. 2014].

\section{Methods}

In this section, we present the developed methods to identify the acquisition problems described in 2.1. The techniques were implemented in the version 7.7.0.471 of MATLAB.

\subsection{Preprocessing}

Firstly a segmentation is applied in order to define the breast area [Méndez et al. 1996]. In this work, this is done through thresholding. When analyzing the characteristics of input images, specifically their gray level, it is observed that the breast region can be delimited through a threshold considering that value $l$ [Sonka et al. 2014], which can be found by observing the image histogram, that are predominantly bimodal (Fig. 2). Since a great number of pixels appear in the histogram dark level, the first mode is 0 .

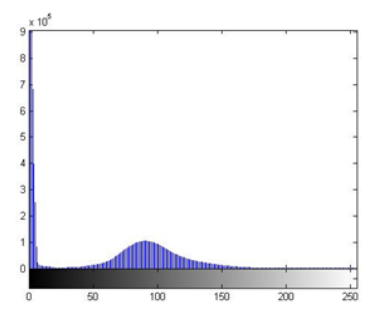

Figura 2. Histogram of the CC mammogram in Fig. 1

That happens because the background in the images is almost uniform, with pixels of very low intensity values. Fig. 3 shows the application of the thresholding with $l=6$ and $l=89$ in the original image, considering a gray scale defined in the $[0,255]$ interval. 


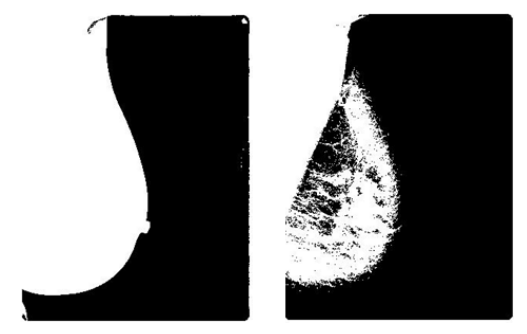

Figura 3. Result of thresholding process with $l=6$ (left) and $l=89$ (right) applied to the MLO mammogram of Fig.1

For pectoral muscle detection the threshold value $l$ was 89. In the other analyzes $l$ was 6 . These values were empirically chosen.

Although the background region is well delimited, there are still some noises present in the images, that can be treated by applying the morphological operations of erosion and dilation [Sonka et al. 2014]. Fig. 4 shows the result obtained by the application of these operators. The structural element used was a 10 pixels side square, completely fulled with white pixels.

After these steps, it is necessary to determine if it is a mammography of a right or left breast. For this a serie of scan lines, i.e. parallel horizontal lines were created, and it is analyzed where they intersect the edge of the image (Fig. 4). Note that, in the case it is a mammography of the left breast, the lines intersect the border are consecutively positioned on the right of the first intersection (considering their horizontal direction values). In the case of the right breast, the straight lines cut the border, present after the first intersection next values on the left of this first point, considering its horizontal direction values.

\subsection{Skeletonization for detection of nipple position}

The technique proposed in this work to check if the nipple is in profile and correctly positioned consists of obtaining the skeleton of the preprocessed image (as presented in Fig. 4). The skeleton characterizes the whole object form and indicates small protuberances in its contour [Sonka et al. 2014]. Any irregularity that does not follow the contour generates ramifications in the skeleton. Therefore, considering the pattern of breasts' shapes on mammograms, such property may allow the identification of the nipple position in the image. Note that if the nipple is not profiled, it is not in the contour region, and it is contained in the inner breast region of the image, so then it have been concealed in the pre-processing step. When the nipple is profiled, its coordinates can be determined by the most extreme branch of the skeleton, considering the horizontal direction defined by the analyzed breast (i.e. the leftmost or rightmost branch).

\subsection{Centered nipple}

As mentioned in the section 2.1, one of the points to be observed in the evaluation of the $\mathrm{CC}$ quality is the central location of the nipple, which defines the breast symmetry in the image. The approach used in this study for this analysis is to considering the nipple position in order to evaluate whether the breast is and when both sides of the nipple are similar, that is, how small is the difference of the areas of either side. Thus, a tolerance value is defined for the subtracted area. Differences greater than the tolerance value determine position mammogram errors. 

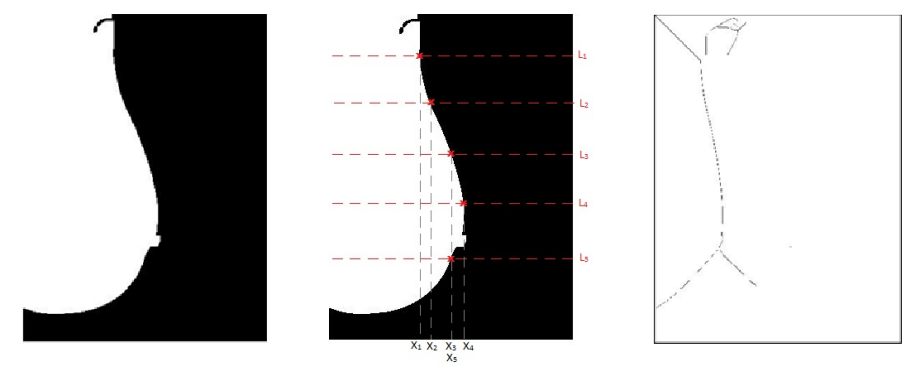

Figura 4. Erosion and dilation morphological operations (left), side determination scan lines (center), and skelotonization (right) of the thresholded MLO mammogram of Fig. 1

\subsection{Hough transform for pectoral muscle identification}

As discussed in the section 2.1, one of the flaws found in MLO mammograms is bad positioning of the pectoral muscle. For this analysis it is necessary to determine the lower edge of the pectoral muscle. In this work, Hough transform with rectilinear elements was used to find such edges [Sonka et al. 2014]. When applying the transform in the MLO image several straight lines would be found, presenting different sizes and angles. Considering MLO mammograms of the left breast, the line that coincides with the pectoral muscle has an inclination that varies around 85 to 60 approx. Considering MLO mammograms of the right breast, the inclination varies around 95 to 120 approx. Lines with that inclination tend to be present smaller size, except for the one which coincides with the pectoral muscle. Thus, the pectoral muscle can be approximated by the segment with the greatest extension that has the inclination in the defined interval.

\section{Results}

The proposed methods were applied in 19 MLO and 17 CC mammogram images. The images are available in the database described in [Silva et al. 2014]. For all the CC images, the patient nipple is visible. For 12 of all 19 MLO images the patient nipple is visible. The pectoral muscle is visible in all MLO images.

In the analysis of $\mathrm{CC}$ views, it was checked if the nipple was centered and if it was in profile. The nipple position was correctly defined by the proposed technique in 13 images, i.e., $76.47 \%$ of the studied cases. The 4 cases in which the nipple was not properly located consists in images with the nipple was not correctly positioned. Considering that the approach proposed in this work uses the location of the profiled nipple to evaluate if the breast is centered, for all these 13 images our method correctly identify if the nipple was centered or not.

In the analysis of MLO views, it was checked if the nipple was in profile and if pectoral muscle was correctly positioned. For all 12 MLO views in which the nipple is visible, its position was correctly found. The cases which the nipple position was not detected correspond to images in which the nipple do not are positioned in profile. Thus, the technique presented correct results for all the MLO views in which the nipple was in the outline. In the analysis of the pectoral muscle position, correct results were found in 12 of 19 cases, i.e., $63.15 \%$. Table 1 shows the results found in this study. 
Tabela 1. Proposed methods results

\begin{tabular}{|c|c|c|c|}
\hline Analysis & Number of evaluated images & View & Correct results \\
\hline Profiled nipple position & 17 & CC & $76.47 \%$ \\
\cline { 2 - 4 } & 12 & MLO & $100.00 \%$ \\
\hline Centered nipple & 17 & CC & $76.47 \%$ \\
\hline Pectoral muscle position & 19 & MLO & $63.15 \%$ \\
\hline
\end{tabular}

\section{Conclusion}

This study evaluated the use of image processing techniques in the identification of acquisition errors in mammographies. The use of morphological operators and thresholding presented good results in the preprocessing step. The results applying the techniques for errors identification led to good results in the analysis performed, especially in the determination of the nipple position.

\section{Referências}

Bassett, L., Hirbawi, I., DeBruhl, N., and Hayes, M. (1993). Mammographic positioning: evaluation from the view box. Radiology, 188(3):803-806.

INCA (2018). Mammograms for radiologists.

Li, Y., Poulos, A., McLean, D., and Rickard, M. (2010). A review of methods of clinical image quality evaluation in mammography. European journal of Radiology, 74(3):e122-e131.

March, M. et al. (2013). Making the diagnosis: A practical guide to breast imaging.

Méndez, A. J., Tahoces, P. G., Lado, M. J., Souto, M., Correa, J., and Vidal, J. J. (1996). Automatic detection of breast border and nipple in digital mammograms. Computer methods and programs in biomedicine, 49(3):253-262.

Popli, M. B., Teotia, R., Narang, M., and Krishna, H. (2014). Breast positioning during mammography: mistakes to be avoided. Breast Cancer: Basic and Clinical Research, 8:BCBCR-S17617.

Silva, L., Saade, D., Sequeiros, G., Silva, A., Paiva, A., Bravo, R., and Conci, A. (2014). A new database for breast research with infrared image. Journal of Medical Imaging and Health Informatics, 4(1):92-100.

Sonka, M., Hlavac, V., and Boyle, R. (2014). Image processing, analysis, and machine vision. Cengage Learning. 